between fullerene and water was modeled by a 60 -site Lennard-Jones potential. The TIP5P model was used to account for interactions between water molecules. Using the NPT ensemble (the number of particles, the pressure, and the temperature were kept constant), the researchers generated molecular conformations using a standard Monte Carlo technique-a stochastic approach that relies on random number generation. Density functional theory calculations were performed on the fullerene and the first hydration shell for only statistically significant conformations. The bandgap of the hydrated system-calculated as the energy difference between the highest occupied and lowest unoccupied molecular orbitals (HOMO and LUMO) averaged over all statistically significant conformations-is $1.1 \mathrm{eV}$. For the unsolvated system, the HOMO-LUMO gap was found to be $1.9 \mathrm{eV}$, in good agreement with the experimental value measured in vacuum. The computational method therefore correctly predicts the red shift in the bandgap resulting from hydration, according to the researchers. An analysis of the density of states for the isolated fullerene at $0 \mathrm{~K}$ and for the hydrated fullerene at $298 \mathrm{~K}$ showed that the red shift of $0.8 \mathrm{eV}$ in the bandgap is caused mainly by structural fluctuations of the hydrating water, not by dielectric screening, which is not expected to substantially redistribute charge on the fullerene surface, said the researchers. The energy difference between the HOMO and LUMO of $\mathrm{C}_{60}$ in the presence of water gives a small average shift of ca. $0.1 \mathrm{eV}$ as a possible solvent effect on the bandgap of the solute. The researchers said that their "procedure avoids the lack of dispersion energy inherent to $a b$ initio molecular dynamics simulations based on DFT," and that their study "is the first theoretical investigation of the finite temperature impact on the electronic structure of the hydrated $\mathrm{C}_{60}$ fullerene using first principles DFT calculations."

SteVEn Trohalaki

\section{Biaxially Stretchable "Wavy" Silicon Nanomembranes on Elastomeric Supports Fabricated}

Y. Huang and J.A. Rogers from University of Illinois at Urbana-Champaign, H. Jiang from Arizona State University, and their colleagues have reported twodimensionally buckled, or "wavy," single crystalline silicon nanomembranes on elastomeric supports with full biaxial stretchability. As described in the June issue of Nano Letters (p. 1655; DOI: 10.1021/ nl0706244), the fabrication protocols start with silicon-on-insulator (SOI) wafers.
A square array of holes ( 2.5 $\mu \mathrm{m}$ diameter, and $25 \mu \mathrm{m}$ pitch) was photolithographically patterned in the top silicon of the SOI wafer. The exposed silicon was removed by reactive ion etching. Immersion of the etched samples in concentrated hydrofluoric acid removed the buried silicon dioxide layer followed by an acetone wash to remove the photoresist. Separately, prepolymers of poly(dimethylsiloxane) (PDMS) were cast and cured against polished silicon wafers to generate flat, elastomeric substrates. Ozone exposure transformed the hydrophobic PDMS surface to a hydrophilic state. Heating in a convection oven at $70-180^{\circ} \mathrm{C}$ induced isotropic thermal expansion in the elastomeric substrate. Contact with the preprocessed SOI wafer and subsequent peeloff transferred the entire nanomembrane to the PDMS. Continued heating facilitated strong adhesion between the membrane and the PDMS. Upon cooling to room temperature, the thermally induced prestrain was released leading to the spontaneous formation of wavy structures in the silicon nanomembrane.

Two-dimensional herringbone layouts were typically observed in the inner regions of the membrane which became somewhat disordered toward the center. Near the edges, however, one-dimensional periodic waves were observed. Optical micrographs acquired during different stages revealed that one-dimensional waves were initially formed over large areas which ultimately evolved into compact herringbone layouts upon cooling.

Stretchability in the nanomembrane was revealed upon uniaxial tensile stretching in various in-plane directions. Upon release of the applied strain, the unbuckled waves revert to the wavy herringbone layout similar to the original, even through multiple stretching cycles. Thermally induced tensile strains enabled examination of biaxial stretchability of the wavy nanomembranes. The herringbone waves at $25^{\circ} \mathrm{C}$ slowly disappeared upon heating to $100^{\circ} \mathrm{C}$, only to be recovered completely upon cooling, the researchers said.

Besides the square membranes described so far, the researchers also investigated other membrane geometries including circles, ovals, hexagons, and triangles. To demonstrate application of biaxial stretchability in electronic devices, a complex array of squares $(100 \mu \mathrm{m} \times 100 \mu \mathrm{m})$ interconnected by ribbons $(30 \mu \mathrm{m} \times 150 \mu \mathrm{m})$ was fabricated. Any applied strain was primarily accommodated by the wavy structures in the ribbons, leaving the square islands mostly undeformed through different strain regimes. The researchers said that such functionality of the buckled nanomembranes of silicon may find widespread use in high-performance electronics where two-dimensional stretchability is desirable during use or installation.

SOMA CHATTOPADHYAY

\section{Nanosized Light Source Offers Possibilities in Bio-Imaging}

A bio-friendly, nanosized light source capable of emitting coherent light across the visible spectrum has been invented by a team of researchers from Lawrence Berkeley National Laboratory (LBNL), the University of California at Berkeley, and the Materials Laboratories at Sony Corporation, Japan. Among the many potential applications of this nanosized light source, once the technology is refined, are single cell endoscopy and other forms of subwavelength bio-imaging, integrated circuitry for nanophotonic technology, and advanced methods of cyber cryptography.

In the June 28 issue of Nature (p. 1098; DOI: 10.1038/nature05921), P. Yang and J. Liphardt, who hold joint appointments in both LBNL and UC-Berkeley, and their colleagues describe a technique in which nanowires of potassium niobate were synthesized in a special hot water solution and separated using ultrasound. The wires were highly uniform in size, several microns long, but only about $50 \mathrm{~nm}$ in diameter. A light beam from an infrared laser was used to create an optical trap that allowed individual nanowires to be grabbed and manipulated. Because of potassium niobate's optical properties, this same beam of infrared laser light also served as an optical pump, causing the nanowires to emit visible light whose color could be selected. In a demonstration of the technique's potential, these nanowire light sources were used to generate fluorescence from specially treated beads.

"Our potassium niobate nanowires have diameters that are substantially below the wavelengths of visible light," said Yang. "They also have excellent electronic and optical properties, and low toxicity, plus they are chemically stable at room temperatures. This makes them ideal for subwavelength laser and imaging technology."

Liphardt said, "In microscopy, the general rule has always been that you can look at an object or you can touch it. With our nanowire light source technology, we combine both these capabilities in a single device. This opens up the possibility of being able to manipulate a specimen as you visualize it."

Central to the success of the nanowire light source are the exceptional nonlinear optical properties of potassium niobate, 
according to the researchers. These nonlinear properties enable the frequencies of the incoming infrared light to be doubled or mixed, through techniques known respectively as second harmonic generation (SHG) or sum frequency generation (SFG), before being emitted as visible light. The result is light that is tunable as well as coherent, which fulfills a technological requirement that has posed a major challenge for both photo-imaging and photo-detection in subwavelength optics.

Bio-imaging may be the field in which this nanowire light source technology has its biggest impact. Optical or visible light microscopy remains at the forefront of biological research because it allows scientists to study living cells and tissues. However, whereas the resolution of optical microscopy is limited by diffraction, through subwavelength techniques it becomes possible to visualize features smaller than visible light wavelengths. For biology, this brings normally invisible subcellular structures into view.

"We hypothesized that a single potassium niobate nanowire would, when optically trapped, be able to double the frequency of the trapping light and then waveguide this locally generated light to its ends, thereby enabling the development of a novel form of scanning light microscopy," said Liphardt. "In addition to demonstrating this scanning transmission mode, we also demonstrated a fluorescence mode."

When a nanowire light source was touched to a fluorescent bead, the bead emitted a distinct orange fluorescence at the contact point. When the nanowire was removed, the orange fluorescence was immediately reduced 80-fold, confirming that the nanowire was the predominant source of fluorescent excitation.

"The work shows that we can create and operate coherent bio-friendly, nanoscale light sources in liquid environments and use them for subwavelength imaging," said Yang.

\section{Multipass Extrusion Creates Microchanneled YSZ/LSM Solid- Oxide Fuel Cell}

In the June 2007 issue of the Journal of the American Ceramic Society (p. 1921, DOI: 10.1111/j.1551-2916.2007.01582.x), three researchers from South Korea have reported a novel extrusion process to create a solid oxide fuel cell containing scores of microtubular cells. Researchers B.-T. Lee of Soonchunhyang University, A.H.M. Esfakur Rahman of Kongju National University, and J.-H. Kim of the

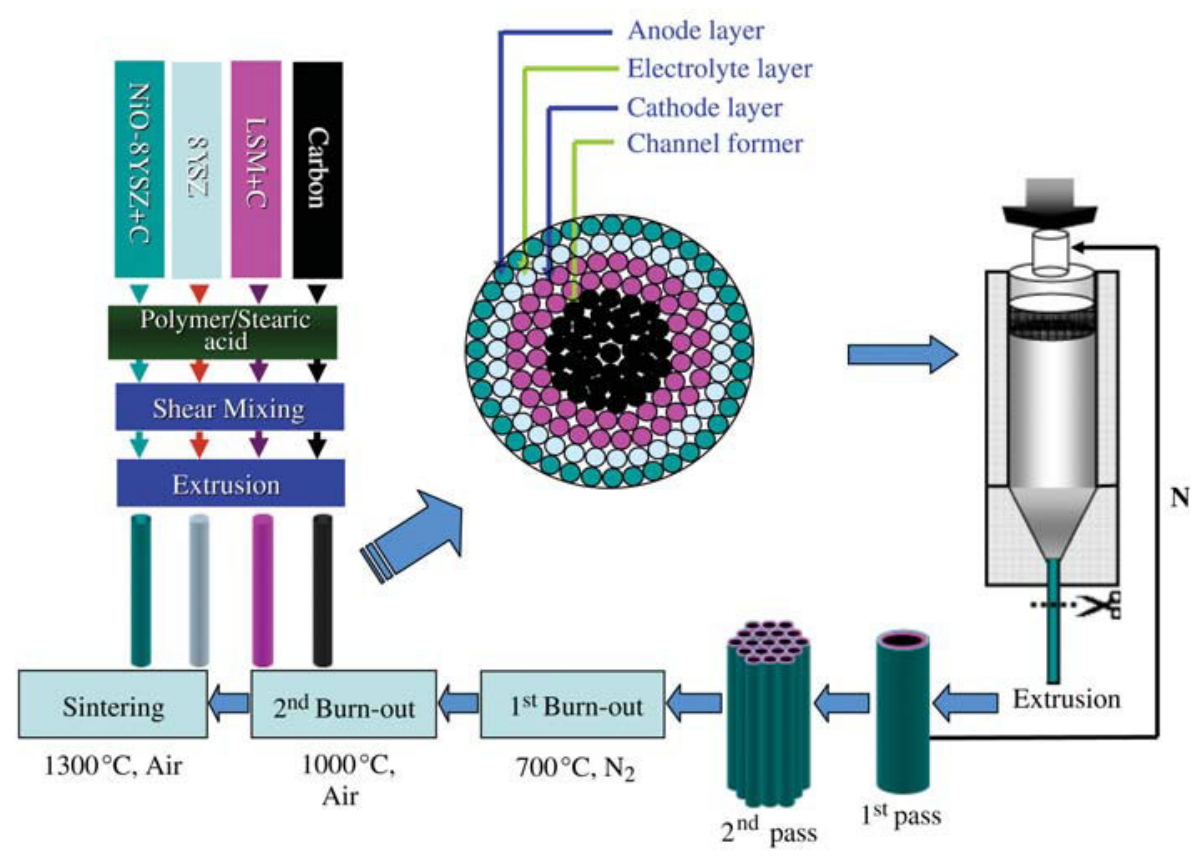

Figure 1. The process for fuel cell fabrication begins at left with homogenous mixing of materials and extrusion into filaments. Layered filaments then form the feedstock for single cell bodies in the first pass extrusion. Many single cells extruded together generate the final green body in the second pass. Heat treatments complete the fabrication process.

Reproduced with permission from Journal of the American Ceramic Society 90 (6) (June 2007) p. 1921; DOI: 10.1111/j.1551-2916.2007.01582.x. (C2007 American Ceramic Society.

Technical Research Laboratories at POSCO first extruded single cells with each containing a central carbon fiber core surrounded by three subsequent layers of filaments: a $\mathrm{La}_{0.8} \mathrm{Sr}_{0.2} \mathrm{MnO}_{3}$ (LSM) cathode layer, an $8 \mathrm{~mol} \%$ yttria-stabilized zirconia (8YSZ) electrolyte layer, and a NiO-8YSZ anode layer (see Figure 1). Grouping 61 single cells, the researchers extruded the group together into a single green body, fired the material, and obtained a fuel cell with an outside diameter of $2.7 \mathrm{~mm}$ and 61 interior microchannels with a diameter of $150 \mathrm{~mm}$. Fuel cells small in size but with high volumetric power density have potential for portable power generation in devices like laptop computers and cell phones.

The multipass extrusion process is novel because of its success in combining single tubular cells and extruding them together to create a fuel cell green body with the original pre-fired single cell diameter $(3.5 \mathrm{~mm})$. The researchers chose their materials to maximize conductivity while maintaining mechanical stability in the fuel cell. The relative composition of $\mathrm{NiO}$ and $8 Y S Z$ in the anode maximized conductivity with the addition of $\mathrm{Ni}$ and minimized thermal mismatch between the anode and the 8YSZ electrolyte layer. The starting electrode mixtures contained activated carbon particles.

The green body materials required three rounds of heat treatment. The first fire burned out polymer binders and lubricants. The second fire burned out the central carbon fibers, leaving hollow microchannels to function as fuel passageways, and burned out carbon particles in the electrodes, leaving open pores to facilitate electrochemical reactions at triple phase boundaries. The third heat treatment sintered the materials and created a dense electrolyte and networked anode layers.

Optical micrographs showed that the electrode and electrolyte layers maintained their integrity after the extrusion processes. Scanning electron micrographs and x-ray diffraction profiles further demonstrated that the electrolyte was dense and crack free and that no reaction compounds existed between the electrode-electrolyte layers in the final fuel cell.

Only limited by the diameter of the extrusion die, the process could create fuel cells with many more microchannels, which would increase the power density, according to the researchers.

ASHLEY PREDITH 